\title{
Evolution of substrate specificity in the Nucleobase- Ascorbate Transporter (NAT) protein family
}

\author{
Anezia Kourkoulou ${ }^{1, \#}$, Alexandros A. Pittis ${ }^{2, \#}$ and George Diallinas ${ }^{1, *}$ \\ ${ }^{1}$ Department of Biology, National and Kapodistrian University of Athens, Panepistimioupolis, Athens 15784, Greece. \\ ${ }^{2}$ Department of Botany, University of British Columbia, Vancouver, BC, Canada. \\ \# Equal contribution. \\ * Corresponding Author: \\ George Diallinas, Department of Biology, National and Kapodistrian University of Athens, Panepistimioupolis, Athens 15784, Greece; \\ Tel. +302107274649; E-mail: diallina@biol.uoa.gr
}

\begin{abstract}
L-ascorbic acid (vitamin C) is an essential metabolite in animals and plants due to its role as an enzyme co-factor and antioxidant activity. In most eukaryotic organisms, L-ascorbate is biosynthesized enzymatically, but in several major groups, including the primate suborder Haplorhini, this ability is lost due to gene truncations in the gene coding for L-gulonolactone oxidase. Specific ascorbate transporters (SVCTs) have been characterized only in mammals and shown to be essential for life. These belong to an extensively studied transporter family, called Nucleobase-Ascorbate Transporters (NAT). The prototypic member of this family, and one of the most extensively studied eukaryotic transporters, is UapA, a uric acid-xanthine/ $\mathrm{H}^{+}$symporter in the fungus Aspergillus nidulans. Here, we investigate molecular aspects of NAT substrate specificity and address the evolution of ascorbate transporters apparently from ancestral nucleobase transporters. We present a phylogenetic analysis, identifying a distinct NAT clade that includes all known L-ascorbate transporters. This clade includes homologues only from vertebrates, and has no members in non-vertebrate or microbial eukaryotes, plants or prokaryotes. Additionally, we identify within the substrate-binding site of NATs a differentially conserved motif, which we propose is critical for nucleobase versus ascorbate recognition. This conclusion is supported by the amino acid composition of this motif in distinct phylogenetic clades and mutational analysis in the UapA transporter. Together with evidence obtained herein that UapA can recognize with extremely low affinity L-ascorbate, our results support that ascorbate-specific NATs evolved by optimization of a sub-function of ancestral nucleobase transporters.
\end{abstract}

\author{
doi: $10.15698 / \mathrm{mic} 2018.06 .636$ \\ Received originally: 28.01.2018; \\ In revised form: 14.03.2018, \\ Accepted 14.03.2018, \\ Published 22.03.2018.
}

Keywords: transporters, L-ascorbate, nucleobase, Aspergillus nidulans, primates.

\section{Abbreviations:}

$A P R$ - ancestral protein resurrection,

NAT-Nucleobase-Ascorbate

Transporter.

\section{INTRODUCTION}

Gene duplication and divergence is a major mechanism of evolutionary novelty, providing protein families with an expanded repertoire of activities and novel specificities. The classic view from Susumu Ohno [1-4] is that duplication creates redundancy, and redundancy provides the material for novelty. Different evolutionary scenarios, such as dosage effects, neo-functionalization or subfunctionalization, might rationalize preservation of gene duplicates [5-10]. The long-term outcome of neofunctionalization is that one copy retains the original function of the gene, while the second copy, free from selective pressure, explores novel functions. The major criticism of this model is the high likelihood of loss of functionality of the gene product before acquiring a new function, as acquiring functional novelty often needs several intermediate evolutionary steps some of which might introduce deleterious mutations. Novelty might evolve via promiscuous side-activities or sub-functions of proteins, which are optimized in the course of evolution and under specific selective pressures. This model puts emphasis on the multifunctional pre-duplication state of the evolving genes and introduces the possibility that positive selection pressure might drive evolution after gene duplication event. A major issue in the evolution of proteins that remains little an- 
swered is how prominent functional changes, which would require the accumulation of multiple serial mutations, take place over the course of time. If many steps in a defined order were necessary for a dramatic functional or specificity change, what would have been the function of 'intermediate' proteins? Additional questions remain as to how protein evolution proceeds after gene duplications. Does protein evolution proceed in small steps or in large steps? Is evolution reversible? How does complexity evolve? Is functional specificity determined solely by the substrate or effector binding-site? $[7,10,11]$. One way to approach these questions is to reproduce protein evolution in the laboratory [12]. This can be achieved by systematic rational or random mutagenesis and DNA shuffling of specific genes, given a functional assay is designed to follow functional modification, or more efficiently, by direct genetic selection or genetic screens using model microorganisms. What is emerging from these approaches of directed evolution is that functional evolution necessitates conservation of sufficient protein stability, as conformational epistasis might suppress several routes of neofunctionalization [13].

A "horizontal" or phylogenetic approach of molecular and functional comparison of homologous proteins is also a powerful approach to predict protein functions and how these might have evolved. However, this in silico approach, by itself, can be misleading as in several protein families, function and mostly specificity cannot be predicted due to overlapping divergent and convergent evolutionary events. Over the last decade, "ancestral protein resurrection" (APR) has developed as a novel strategy to reveal the mechanisms and dynamics of protein evolution [14]. APR gives access to a protein's lost properties that may have transiently arisen over evolutionary time, which in turn can be used to infer the potential selection pressures that resulted in present-day sequences. Thus, APR can identify the causative mutation that resulted in protein neofunctionalization after gene duplication. APR has confirmed that conformational epistasis limits the pathways of functional evolution as neutral mutations may act as ratchets to modification, and additionally supported that evolution seems to proceed from promiscuity to increased specificity, usually via loss-of-function mutations [15].

A still unexplored aspect in protein evolution concerns whether transmembrane proteins, especially those of eukaryotic cells, evolve at similar rates and under similar evolutionary constraints as soluble proteins. This question arises from a number of peculiarities of transmembrane proteins, as for example, their co-translational folding and translocation in the ER membrane, the very complex and highly regulated subcellular traffic, the highly controlled ubiquitination-dependent turnover routes, and their dynamic interaction with specific lipids [16-18]. These special aspects of transmembrane proteins should in principle impose additional constraints or alternative selective pressures for evolution.

Here, we ask how mammalian L-ascorbate (vitamin C) transporters might have evolved from, apparently, ances- tral nucleobase transporters. This question is based on the fact that members of the so-called Nucleobase Ascorbate Transporter (NATs) family [19-23] are highly specific for either nucleobases or L-ascorbate (see also later). This shift in substrate specificity is a dramatic one as these solutes have very different chemical formulas and properties and probably necessitate prominent changes in the architecture of the substrate-binding site and substrate translocation trajectory in the relevant NATs. Our results support a scenario that ascorbate-specific NATs evolved by optimization of a sub-function of ancestral nucleobase transporters.

\section{RESULTS AND DISCUSSION}

NATs are functionally classified into two distinct specificity groups: nucleobase versus L-ascorbate transporters

More than 24 members of the NAT family have been functionally characterized via direct assessment of their transport activities or indirectly via genetic and physiological studies [19-23] (Table 1). The most extensively studied NATs come from Escherichia coli or the filamentous ascomycete Aspergillus nidulans. The rest are homologues from other bacteria (Bacillus subtilis, Sinorhizobium meliloti), ascomycetes (Aspergillus fumigatus, Aspergillus brasiliensis, Candida albicans) or plants (maize, Arabidopsis thaliana). All bacterial NATs are $\mathrm{H}^{+}$symporters highly specific for either uracil or xanthine or uric acid. The fungal and plant members are $\mathrm{H}^{+}$symporters specific either xanthine-uric acid, or for adenine-guanine-hypoxanthine-uracil (only in plants) [19-25]. All these NATs exhibit relatively high affinity for their physiological substrates (at the low $\mu \mathrm{M}$ range), but can also recognize with lower affinity several nucleobase analogues. Notably, the relatively specific xanthineuric acid NATs of fungi can be genetically converted, by single or limited missense mutations, to become broadspecificity promiscuous nucleobase transporters [26-29]. However, no microbial or plant, wild-type or mutant NAT, is capable of transporting L-ascorbate.

In sharp contrast to the microbial and plant proteins, most functionally characterized mammalian NATs, coming from mouse, rat, wild boar or humans, called SVCT1 or SVCT2 (Sodium-dependent Vitamin C Transporters; SLC23 family members), are highly specific for L-ascorbate/ $\mathrm{Na}^{+}$ [23]. However, there is a case reported of a mammalian (rat) NAT transporter, called rSNBT1, which is specific for nucleobases, rather than L-ascorbate [31]. Interestingly, the genes encoding orthologues of rSNBT1 in most primates (called SVCT4) are truncated pseudogenes. A third distinct homologue of NATs (SVCT3) identified in mammals seems unrelated to either nucleobase or L-ascorbate transport [23]. Thus, the emerging view is that all NATs in microorganisms and plants are nucleobase-specific, whereas mammals have both nucleobase and L-ascorbate NATs, but primates have lost via gene truncation the nucleobase-specific ones. However, this picture is little supported by the limited number and biased selection of functionally characterized NATs. 
Two members of the NAT family have been crystallized. These are the UraA uracil transporter [32] of E. coli and the UapA uric acid-xanthine transporter of $A$. nidulans [33]. Both proteins form tight dimers, the formation of which is essential for transport activity [33-35]. The UraA and UapA monomers are made of mostly $\alpha$-helical segments (TMS) characterized by a 7+7-helix inverted repeat [30]. Interestingly, the same topology is also found in transporters with

Table 1. Characteristics of biochemically characterized NATs.

\begin{tabular}{|c|c|c|c|c|c|c|c|c|}
\hline Transporter name & Origin & $\begin{array}{l}\text { Physiological } \\
\text { substrate }\end{array}$ & $\begin{array}{c}\text { Other } \\
\text { substrates }\end{array}$ & $\begin{array}{l}\text { Cation } \\
\text { symport }\end{array}$ & $\begin{array}{l}\text { Known } \\
\text { structure }\end{array}$ & $\begin{array}{c}\text { Q-H } \\
\text { motif } \\
\text { (TMS1) } \\
\text { Substrate } \\
\text { transport }\end{array}$ & $\begin{array}{l}\text { ExxGD } \\
\text { motif } \\
\text { (TMS8) } \\
\text { Substrate } \\
\text { bind \& } \\
\text { transport }\end{array}$ & $\begin{array}{l}\text { NAT signature motif } \\
\text { (TMS10) } \\
\text { Substrate bind \& } \\
\text { transport \& specificity }\end{array}$ \\
\hline UraA & E. coli & uracil & 5-FU & $\mathrm{H}^{+}$ & $\begin{array}{l}\text { yes/dimer } \\
\text { bound to } \\
\text { uracil }\end{array}$ & yes & $\begin{array}{l}\text { yes } \\
\text { (ExxGH) }\end{array}$ & T-Y-G-E-NxGxxxxTG \\
\hline XanQ (YgfO) & E. coli & xanthine & $\begin{array}{l}\text { xanthine } \\
\text { analogues }\end{array}$ & $\mathrm{H}^{+}$ & $*$ & $\begin{array}{l}\text { yes } \\
\text { (T-H) }\end{array}$ & yes & $\begin{array}{l}\text { T-F-A-Q- } \\
\text { NxGxxxxTG }\end{array}$ \\
\hline XanP (YicE) & E. coli & $\begin{array}{l}\text { Xanthine } \\
\text { low-capacity }\end{array}$ & $?$ & $\mathrm{H}^{+}$ & * & yes & yes & $\begin{array}{l}\text { C-F-G-Q- } \\
\text { NxGxxxxTG }\end{array}$ \\
\hline UacT (YgfU) & E. coli & $\begin{array}{l}\text { uric acid } \\
\text { low-affinity }\end{array}$ & $\begin{array}{l}\text { xanthine } \\
\text { (very low } \\
\text { capacity) }\end{array}$ & $\mathrm{H}^{+}$ & * & yes & $\begin{array}{l}\text { yes/no } \\
\text { ExxGM }\end{array}$ & $\begin{array}{l}\text { S-F-S-Q- } \\
\text { NxGxxxxTG }\end{array}$ \\
\hline UapA & A.nidulans & $\begin{array}{l}\text { uric acid- } \\
\text { xanthine }\end{array}$ & $\begin{array}{l}\text { uracil, } \\
\text { xanthine-uric } \\
\text { acid } \\
\text { analogues } \\
\text { oxypurinol, } \\
\text { allopurinol }\end{array}$ & $\mathrm{H}^{+}$ & $\begin{array}{l}\text { yes/ } \\
\text { dimer } \\
\text { bound to } \\
\text { xanthine }\end{array}$ & yes & yes & T-F-A-Q-NxGxxxxTR \\
\hline UapC & $\begin{array}{l}\text { A.nidulans } \\
\text { A.fumigatus }\end{array}$ & $\begin{array}{l}\text { xanthine - } \\
\text { uric acid }\end{array}$ & $\begin{array}{l}\text { uracil, } \\
\text { xanthine-uric } \\
\text { acid } \\
\text { analogues } \\
\text { oxypurinol }\end{array}$ & $\mathrm{H}^{+}$ & * & yes & yes & $\begin{array}{l}\text { V-F-A-Q- } \\
\text { NxGxxxxTR/K }\end{array}$ \\
\hline Xut1 & C. albicans & $\begin{array}{l}\text { xanthine - } \\
\text { uric acid }\end{array}$ & $\begin{array}{l}\text { uracil, } \\
\text { xanthine-uric } \\
\text { acid } \\
\text { analogues } \\
\text { oxypurinol }\end{array}$ & $\mathrm{H}^{+}$ & * & yes & yes & $\begin{array}{l}\text { V-F-A-Q- } \\
\text { NxGxxxxTK }\end{array}$ \\
\hline Lpe1 & Zea mays & $\begin{array}{l}\text { uric acid- } \\
\text { xanthine }\end{array}$ & & $\mathrm{H}^{+}$ & $*$ & yes & yes & A-S-V-E-NxGxxxxTG \\
\hline Nat3 & A. thaliana & $\begin{array}{l}\text { adenine, } \\
\text { hypoxanthine } \\
\text { guanine, uracil }\end{array}$ & $?$ & $\mathrm{H}^{+}$ & * & yes & yes & A-S-V-E-NxGxxxxTG \\
\hline Nat12 & A. thaliana & $\begin{array}{l}\text { adenine, } \\
\text { hypoxanthine } \\
\text { guanine, uracil }\end{array}$ & $?$ & $\mathrm{H}^{+}$ & * & yes & yes & T-L-T-E-NxGxxxxTG \\
\hline $\begin{array}{l}\text { rSNBT1 } \\
\text { (SVCT4) }\end{array}$ & $\begin{array}{l}\text { Rat, mouse } \\
\text { predominantly in } \\
\text { small intestine } \\
\text { pseudogene in } \\
\text { primates }\end{array}$ & $\begin{array}{l}\text { hypoxanthine, } \\
\text { xanthine } \\
\text { guanine, uracil, } \\
\text { thymine }\end{array}$ & $\begin{array}{l}\text { 5-FU, } \\
\text { oxypurinol }\end{array}$ & $\mathrm{Na}^{+}$ & * & yes & yes & S-Y-S-E-NxGxxxxTR \\
\hline SVCT1 & $\begin{array}{l}\text { Rat, mouse, } \\
\text { human } \\
\text { Epithelia kidney, } \\
\text { intestines, liver, } \\
\text { lung, skin }\end{array}$ & L-ascorbate & - & $\begin{array}{l}2 \mathrm{Na}^{+} \\
\text {(need of } \mathrm{Ca} 2+ \\
\text { or } \mathrm{Mg}^{2+} \text { ) }\end{array}$ & * & yes & yes & S-S-S-P-NxGxxxxTK \\
\hline SVCT2 & $\begin{array}{l}\text { Rat, mouse, } \\
\text { human } \\
\text { Widespread, } \\
\text { including Brain }\end{array}$ & L-ascorbate & - & $\begin{array}{l}2 \mathrm{Na}^{+} \\
\text {(need of } \mathrm{Ca} 2+ \\
\text { or } \mathrm{Mg} 2+\text { ) }\end{array}$ & * & yes & yes & S-S-S-P-NxGxxxxTK \\
\hline SVCT3 & $\begin{array}{l}\text { Vertebrates } \\
\text { predominantly in } \\
\text { kidney }\end{array}$ & $?$ & $?$ & $\mathrm{Na}^{+}$ & * & yes & $\begin{array}{l}\text { no } \\
\text { (SxxGC) }\end{array}$ & S-S-F-P-NxGxxxxTG \\
\hline
\end{tabular}

All NATs shown are high-affinity transporters recognizing their physiological substrate at the low $\mu \mathrm{M}$ range, except UacT which is low affinity transporter. NATs marked with an $\left(^{*}\right)$ can be modeled, with $100 \%$ probability, on the crystal structure of UapA or UraA conforming to the 7+7 inverted repeat fold [30]. 5-FU is 5-fluorouracil. Consensus sequences of three motifs involved in function and specificity are shown. Notice that the most significant differences in the NAT signature motif of nucleobase versus L-ascorbate transporters concern substitutions of the aromatic Phe/Tyr at position 2 and of the polar Gln/Glu with a Pro. ?, not tested. "S. Frillingos pers. com. 
very little primary amino acid sequence similarity with NATs, such as the AzgA-like purine transporters [36] the plant boron transporter Bor1 [37], the human Band3 anion exchanger [38], or members of SulP transporter family [39]. All these are homodimeric transporters which seem to function via the so-called "elevator mechanism" of transport [40].

\section{L-ascorbate transporters belong to a phylogenetically distinct NAT clade}

We performed an extensive phylogenetic analysis of the NAT family using different datasets and methodologies. NAT sequences used for the analysis are, with very few exceptions, made of 414-650 amino acid residues in length. Selected NAT representatives from all major taxa were also tested, using the HHpred modeling program (https://toolkit.tuebingen.mpg.de), and shown to conform to the 7+7-helix inverted repeat structure of UapA (analysis not shown). Initially we reconstructed a phylogenetic tree using identified NAT homologues in all domains of life, from archaea and bacteria, to fungi, algae, plants and animals (see later). Prominent evolutionary gene-losses of NATs are identified several pathogenic protozoa (e.g. Trypanosoma, Plasmodium, Leishmania, Giardia, etc.) and in mesozoa, in several basal fungi including Microsporidia, Cryptomycota and Blastocladiomycota, as well as, in several Saccharomycetales, after the whole genome duplication. Interestingly, NAT loss in Saccharomycetales is concomitant with gene loss of uric acid oxidase, formation of an allantoin utilization cluster [41] and the "generation" of xanthine dioxygenase (Claudio Scazzocchio, unpublished observations). Other NAT losses in the animal clade are detected in basal Metazoa, such as the Porifera (sponges), some Ctenophora (Eumetazoa), some jaw worms (Gnathostomulida and Xenacoelomorpha), and among earlydiverging fish (jawless fish, lampreys or hagfish).

Figure 1 and Supplementary Figures S1 and S2 highlight our major findings in respect to the distribution of NAT sequences. The first clear observation is that there is a primary evolutionary split between microbial (homologues in archeae, bacteria, algae and fungi, collectively called UapA-like) and plant-animal NATs (Figure 1a). In the microbial/UapA-like group, there are distinct sub-clades for several bacterial groups, archaea, algae and fungi (Supplementary Figure S1). A handful of protists, such as Dictyostelium discoideum, are also clustered with specific bacterial clades, suggesting that these might be the products of horizontal gene transfers. A second basic observation is that in the plant-animal clade (SVCT clade), the plants form a clear monophyletic branch including dicots and monocots, as well as, bryophytes (e.g. Physcomitrella patens) and some green algae (e.g. Klebsormidium nitens) (Figure 1a and Supplementary Figure S2). The animal clade includes several distinct branches. These however can be grouped in four major branches, which herein we named SVCT1, SVCT2, SVCT3 and SVCT4, based on the terminology of the mammalian homologues (Figure 1 and Supplementary Figure S2). The amino acid sequence identity of members of the microbial, plant or animal clades is $\sim 30-92 \%$, whereas the identity between microbial-plant, microbial-animal or plant-animal NATs is $\sim 24-33 \%$.

To gain deeper insights into the evolution of the plant and animal - specific - SVCT clade, we focused on sequences from this clade and deepened our taxon sampling. We used HMMER [43] and the NAT Pfam domain (PF00860) to extract 728 NAT sequences from 171 plant and animal fully sequenced genomes (see methods). After reconstructing a phylogenetic tree using these sequences, we detected and extracted only the 674 sequences comprising the SVCT clade (Figure 1a and Supplementary Figure S2). In the animal clade, an "extended" SVCT4 group (> 45\% identity among its members) is widely distributed across Metazoa, and includes members from most vertebrates, nonvertebrates and early diverging animals, as for example, Trichoplax adhaerens (the only extant representative of phylum Placozoa, which is a basal group of multicellular metazoa), Nematostella vectensis (sea anemone belonging to Cnidaria), Ciona intestinalis (sea squirt), Strongylocentrotus purpuratus (sea urchin), Orbicella faveolata (coral), Macrostomum lignano (flatworm), etc. (Supplementary Figure S2). Most SVCT4-positive species include a single SVCT4 homologue, but there is also significant genusspecific expansion of the group, apparently by gene duplications (e.g. 6-14 paralogues in Caenorhabditis, 8 in Ciona intestinalis, 5-6 in some fish; Figure 1b). Interestingly, all Haplorhini (anthropoid primates or simians and the tarsier Carlito syrichta) have lost functional SVCT4 homologues, due to gene truncations (i.e. generation of pseudogenes; [23]). "Patchy" SVCT4 loss is detected also in some nonprimate species, but these correspond to genus-specific cases, rather than representing losses in major groups (Figure 1b). Interestingly, SVCT4 loss often coincides with the loss in the ability to synthesize L-ascorbate, although not all known cases that lack this ability lack SVCT4 proteins. Despite the fact that only a single member of the SVCT4 group is functionally characterized (i.e. the rat rSNBT1 nucleobase transporter [31]), the conservation of specific functional motifs present in bacterial, plant or fungal nucleobase transporters, allows us to hypothesize that members of the SVCT4 group are specific for nucleobaserelated substrates (see later).

The SVCT1 and SVCT2 paralogous subfamilies form a separate clade ( $\sim 50 \%$ identity with SVCT4 in the same species) that includes the well-characterized mammalian L-ascorbate transporters. The SVCT1/SVCT2 group appears exclusively in vertebrates and is probably the result of duplication and diversification of an ancestral animal transporter. Most species maintain one member of SVCT1 and SVCT2 (Figure 1b). Thus, a gene duplication event in the vertebrate ancestor seems to have generated the two subfamilies. As the SVCT1 and SVCT2 proteins show high similarity (>68\% identity) and all functionally characterized homologues are L-ascorbate transporters, one can speculate that the specificity of L-ascorbate has evolved in the vertebrate ancestor and was retained in fish and tetrapods (see Fig.1). However, whether all extant members of the SVCT1/SVCT2 group are specific L-ascorbate transporters, 
a

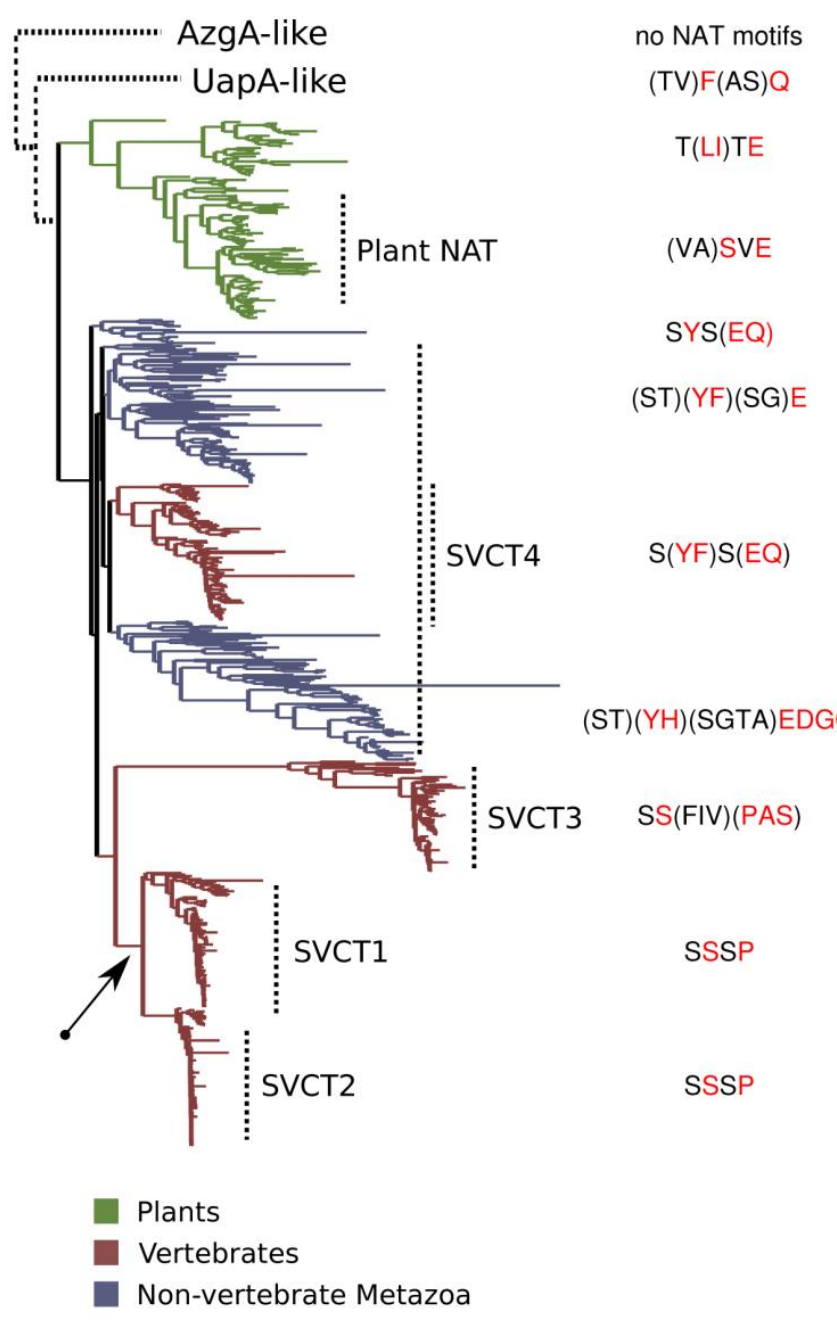

b

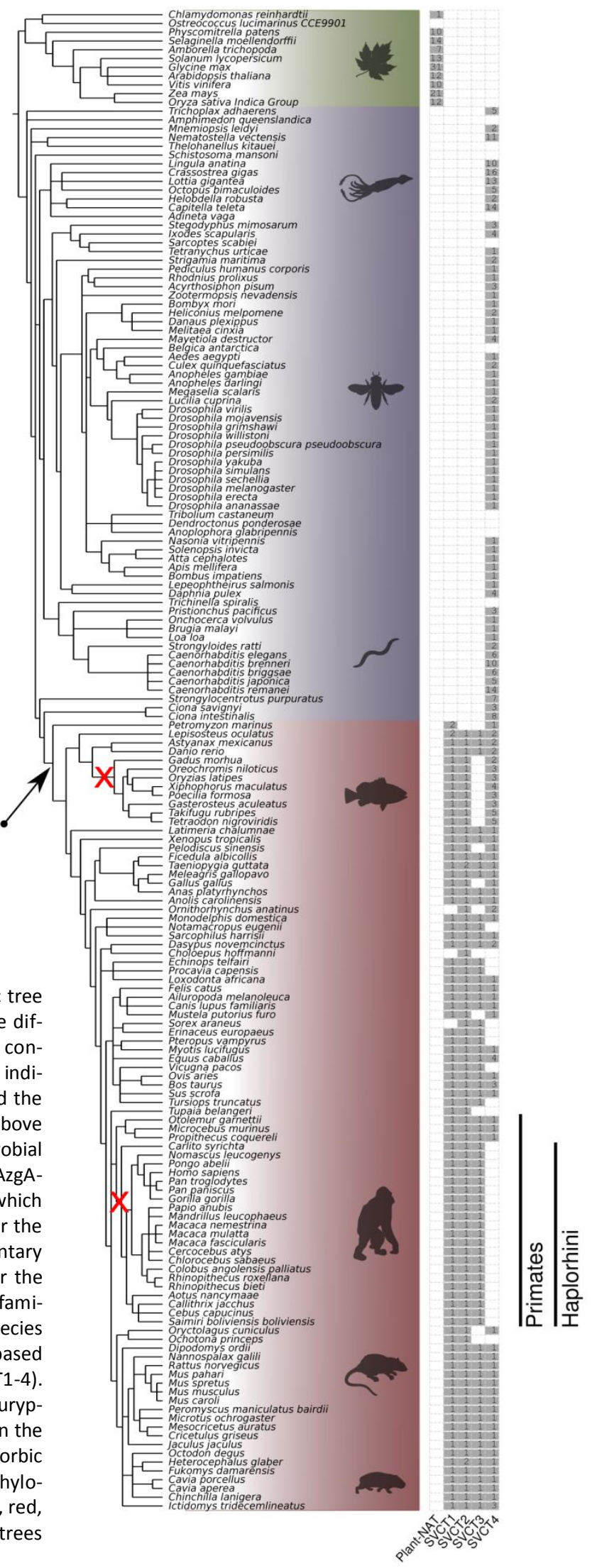

FIGURE 1: Evolution of the SVCT clade in NATs. (a) ML phylogenetic tree of the SVCT clade of NATs and the distinct NAT signature motifs. The different SVCT plant and animal NAT subfamilies, as well as a variable consensus of the four first amino acids in the NAT signature motif, are indicated next to the tree. The tree was midpoint rooted, as shown, and the other two clades, UapA-like and AzgA-like, are placed schematically above with dotted lines. The UapA-like group corresponds to canonical microbial NATs and is shown in more detail in Supplementary Figure S1. The AzgAgroup is used as an out-group of structurally homologous proteins, which however do not conserve the functional motifs of canonical NATs. For the complete version of the plant and animal NATs see also Supplementary Figure S2. (b) Phylogenetic profile of the SVCT clade sub-families for the 171 plant and animal species. The frequency of members in each subfamily is shown next to the NCBI taxonomy tree structure of the 171 species that were searched for NAT domains. The sub-families were defined based on (a) and in reference to the known mammalian transporters (SVCT1-4). Two early losses in two lineages, the SVCT3 in the Teleostean clade Eurypterygii and SVCT4 in the primate suborder Haplorhini, are indicated in the corresponding branches with a red " $\mathrm{x}$ ". The emergence of the L-ascorbic acid transport specificity is indicated with a black arrow both in the phylogenetic tree (a), as well as in the taxonomy tree (b). The colors (green, red, blue) in both (a) and (b) are as indicated in the figure legend. Both trees were visualized using the ETE toolkit [42]. 
or whether some SVCT1/SVCT2 homologues transport nucleobases or other substrates, is not formally known.

Finally, SVCT3-like sequences are found in nearly all tetrapod animals and several, but not all, fish (e.g. the Lepisosteus oculatus, Astyanax mexicanus, Danio rerio, Rhincodon typus; (Figure 1b). Noticeably, SVCT3 has been lost in some lineages including the Acanthomorpha, a very diverse clade of teleost fishes. The SVCT3 proteins are also the most divergent NAT group in the animal clade, as not only show the lowest overall amino acid sequence similarity with the other clades ( $<34 \%$ identity with SVCT1/2 and SVCT4), but additionally do not fully conserve the specific amino acid motifs necessary for function and specificity in "canonical" NATs (see later). This might also explain the fact that its phylogenetic position in the tree was rather unstable across different phylogenetic algorithms and parameters (not shown). Thus, SVCT3 members are very probably transporters of substrates other than nucleobases or ascorbate. This is indeed supported by reports showing that the human hSVCT3 does not transport nucleobases or L-ascorbate [23]. An analogous "non-canonical" (i.e. lacking the motifs necessary for nucleobase or L-ascorbate transport) divergent NAT group, called UapD-like proteins, has recently been described in fungi [44], while other divergent NAT branches, all of unknown function, seem to exist also in prokaryotes (Stathis Frillingos pers. com. and observations not shown herein).

The NAT signature motif is differentially conserved in nucleobase and L-ascorbate transporter clades

Previous genetic, molecular, biochemical and structural studies have established that a small number of amino acid residues are essential or critical for NAT function. Most of these fall within three highly conserved motifs in transmembrane segments TMS1, TMS8 and TMS10. The twoamino acid motif Gln-His in TMS1 is nearly absolutely conserved in all NATs (only a small number of mostly microbial homologues have a Gln-Gln, Thr-His or Gln- $\Phi$ versions, where $\Phi$ is Met or lle) and seems to be involved in dynamic interactions of TMS1-TMS3-TMS10 necessary for transport catalysis [19-22, 43, 44]. The TMS8 motif, conforming to the sequence Glu-X-X-Gly-Asp/His/Gly (in bold the most prominently conserved residues), is involved in interactions with substrates and transport [19-22, 46, 47]. Finally, a longer motif in TMS10, GIn/Glu/Pro-Asn-X-Gly-X-X-X-X-ThrGly/Arg/Lys, historically referred as the NAT signature motif [47], is essential for both substrate recognition and transport activity, as well as, substrate specificity [1922,46-48]. The presence of these three motifs is taken, in the present analysis, as an additional criterion for classifying any uncharacterized transporter as a member of the "canonical" NAT family.

The phylogenetic analysis performed herein confirms the presence and exceptional sequence conservation of these motifs in nearly all NAT clades. More specifically, the motifs in TMS1 and TMS8 are highly conserved in all NATs, except from the highly divergent SVCT3 group, which conserves the TMS1 motif, but lacks the TMS8 motif (observations not shown). The motif in TMS10 exists in all NATs but appears in two major versions, especially if we consider three additional upstream amino acid residues (see Figure $1 \mathrm{a}$ and Supplementary Figure S2). More specifically, in functionally characterized nucleobase-specific NATs and their close homologues it conforms to the sequence Thr/Ser ${ }^{1}$-Phe/Tyr ${ }^{2}$-Ala/Ser ${ }^{3}$-Gln/Glu/Pro ${ }^{4}$-Asn ${ }^{5}$-X-Gly ${ }^{7}$-X-X-X$\mathrm{X}-\mathrm{Thr}^{12}$-Gly/Arg/Lys ${ }^{13}$ (numbers shown are arbitrary). In the L-ascorbate transporter clades (i.e. SVCT1 and SVCT2) this

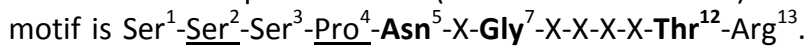
In other words, the most prominent changes, shown underlined, in the two versions of this extended TMS10 motif concern replacements of the aromatic $\mathrm{Phe} / \mathrm{Tyr}^{2}$ and polar $\mathrm{Gln} / \mathrm{Glu}^{4}$ (nucleobase transporters) by $\mathrm{Ser}^{2}$ and $\mathrm{Pro}^{4}$ residues (L-ascorbate transporters). Interestingly, the divergent SVCT3 group has an apparent TMS10 motif more similar to the one found in L-ascorbate transporters rather to that of nucleobase transporters, which also supports its close evolutionary relatedness to them (see Figure 1a).

Based on previous functional [19-22] and recent structural $[32,33,35]$ studies performed in fungal (e.g. UapA) and bacterial (e.g. XanQ) homologues, $\mathrm{Gln} / \mathrm{Glu}^{4}$ is one of the two major NAT residues interacting via a strong bipolar interaction with the nucleobases (the other being the absolutely conserved Glu in the TMS8 motif), while Phe/Tyr ${ }^{2}$ also interacts directly with the nucleobase ring via pi-pi stacking. Most importantly, Phe/Tyr ${ }^{2}$ and Gln/Glu ${ }^{4}$ have proved as key elements in determining the specificity of NATs in respect to different nucleobases. This is best exemplified by specific mutations in UapA, where substitution of $\mathrm{Gln}^{4}$ by $\mathrm{Glu}^{4}$ (e.g. Q408E) converts this relative specific uric acid-xanthine transporter into a promiscuous transporter capable of recognizing hypoxanthine, guanine or uracil [27], whereas replacement of $\mathrm{Phe}^{2}$ by $\mathrm{Tyr}^{2}$ (e.g. F406Y) confers a detectable capacity for hypoxanthine transport [29]. Thus, it becomes apparent that more dramatic substitutions of Phe/Tyr ${ }^{2}$ or $\mathrm{Gln} / \mathrm{Glu}^{4}$, introducing residues as the ones found in L-ascorbate specific NATs (i.e. $\mathrm{Ser}^{2}$ and $\mathrm{Pro}^{4}$ ), are expected to result in even more prominent specificity modifications, and might in fact contribute to the "generation" of L-ascorbate transporters. All other conserved residues in the TMS10 signature motif of NATS, including the L-ascorbate transporters, are known to contribute to the kinetic parameters of transport (Thr/Ser ${ }^{1}$, Ala/Ser ${ }^{3}, \mathrm{Asn}^{5}, \mathrm{Gly}^{7}, \mathrm{Thr}^{12}$ and Gly/Arg/Lys ${ }^{13}$ ), rather than playing a direct role in determining substrate specificity.

\section{The differentially conserved residues in the NAT signature motif are essential for function and specificity}

No mutation, up to date, in any nucleobase-specific NAT, has led to a transporter capable of recognizing L-ascorbate. However, it should be stressed that there has been no rational genetic design to do so, as most functional mutations were designed or genetically selected to change the specificity of NATs in respect to different nucleobases rather than to L-ascorbate. The only exception is the substitution of $\mathrm{Gln}^{4}$ by Pro ${ }^{4}$ in the NAT signature motif of the UapA [47] or XanQ transporters [48]. In this case, introducing a proline residue led to dramatic reduction $(>100$-fold) in nucleobase binding and thus no detectable transport 
activity, while no evidence was obtained for acquisition of L-ascorbate recognition.

To further test whether specific variations of the NAT signature motif might be relevant to L-ascorbate recognition, we constructed and/or functionally analyzed a series of relevant UapA mutants. These mutants correspond to the following amino acid replacements in UapA: Q408P [46], A407S/Q408P, T405S/F406S/A407S and F406S/A407S/Q408P. In other words, we modified the first part of the NAT signature motif from TFAQ to TFA $\underline{P}$, TFSP, SSSQ or TSSP (changes in bold underlined). These mutant versions of UapA were expressed into an appropriate $A$. nidulans strain genetically lacking all endogenous major nucleobase transporters (i.e. UapA, UapC and AzgA) and functionally analyzed in respect to their capacity to transport or bind nucleobases or L-ascorbate, and for proper plasma membrane sorting and stability. Growth tests on purines as sole nitrogen sources can be used as a tool to reveal the functionality of the relative purine transporters. Thus, a control strain expressing a functional UapA (i.e. $\operatorname{uapA}^{+}$), but no other major purine transporter, grows on uric acid or xanthine, but not on other purines. Figure 2a shows that all UapA mutants tested have lost (T405S/F406S/A407S and F406S/A407S/Q408P) or have dramatically reduced (e.g. Q408P and A407S/Q408P) capacity for growth on uric acid or xanthine. This was confirmed by direct measurements of transport of radiolabeled xanthine (Figure $2 \mathrm{~b}$ ). In addition, none of the mutants tested have acquired any capacity for transport of any other purine, nucleoside or nucleobase-related metabolite (Figure 2a and results not shown). As all UapA mutants tested seem to correspond to abolished or reduced transport function, we wanted to exclude that this not due to misfolding, problematic sorting to the plasma membrane or protein instability. For this, we took advantage of the fact that all mutations analyzed are made in a fully functional UapA protein tagged with GFP. In vivo epifluorescence microscopy showed that all mutant versions of UapA analyzed are properly and stably localized in the plasma membrane (right panel in Figure 2a), which in turn confirms that all relevant mutations affect transport function per se.

We additionally tested whether the mutations introduced lead to any growth phenotype that could be related to UapA-dependent L-ascorbate accumulation. Previous results have shown wild-type $A$. nidulans cannot import Lascorbate, at least when this is externally supplied to concentration up to $1 \mathrm{mM}$, while higher concentrations of L-ascorbate (5.6-56.8 $\mathrm{mM}$ or $0.1-1 \%$ ) have a progressively moderate inhibitory effect on wild-type $A$. nidulans growth ([24], and unpublished observations). Figure $2 c$ shows that none of the UapA mutations analyzed, or the total genetic absence of UapA (uapA'), alters the growth phenotype of A. nidulans on L-ascorbate. We also tried to detect cytoplasmic accumulation of externally supplied L-ascorbate in the mutants or a wild-type strain, using HPLC or a biochemical assay, or by measuring the accumulation of radiolabeled L-ascorbate. In all cases, we did not obtain any evidence of L-ascorbate accumulation (results not shown).
Our results suggested that rational modification of the NAT signature motif is not sufficient, by itself, to confer detectable L-ascorbate accumulation. However, the stable and proper cellular expression of the mutants enabled us to use a sensitive in vivo cellular assay, based on substrateelicited endocytosis, as an indirect measure of the ability of different UapA versions to "recognize", and not necessarily transport at a detectable level, L-ascorbate. This assay is based on the fact that UapA is highly sensitive to endocytic turnover triggered by substrate or ligand binding $[49,50]$. Substrate-elicited transporter turnover is generally promoted by activity-dependent alteration of the conformation of a transporter from an outward-facing to a cytoplasm-facing topology, and can be followed by the appearance of fluorescing vacuoles and early endosome due to sorting of GFP-tagged transporters into these organelles $[49,50]$. Based on this, we tested whether L-ascorbate could promote the endocytosis of wild-type or mutant versions of UapA-GFP, as well as, of a control transporter unrelated to NATs, namely FurA-GFP. FurA is a highly specific allantoin transporter that does not recognize either nucleobase or L-ascorbate [51].

Figure $2 \mathrm{~d}$ shows that the presences of $0.1 \% \mathrm{~L}$-ascorbate, for $15 \mathrm{~min}$, promotes significant endocytosis of UapA mutants, but has no similar effect on wild-type UapA or FurA. After prolonged presence of L-ascorbate $(1 \mathrm{~h})$, endocytosis of the UapA mutants becomes more prominent, mostly evident in T405S/F406S/A407S and F406S/A407S/Q408P, but also becomes apparent, albeit at a much lower level, in wild-type UapA. Importantly, no endocytic turnover was detected in FurA, in all conditions tested. Additionally, prolonged incubation with L-ascorbate also elicited total loss of fluorescence, associated with a dramatic change in hyphae morphology, in a significant fraction of the UapA mutants. The L-ascorbate-dependent morphological effect, which was insignificant in strains expressing wild-type UapA or FurA, was reflected as a dramatic increase of the number and size of vacuoles and a reduction in the width of hyphae, two changes often seen under severe cell stress. The ascorbate-dependent effect was mostly observed in the triple mutants F406S/A407S/Q408P (in 55\% hyphae) and T405S/F406S/A407S (in 68\% hyphae) (Figure 2d and 2e), while a similar, albeit reduced, effect is seen in A407S/Q408P (40\%) and Q408P (27\%) (see Figure 2d and $2 \mathrm{e})$. Given that the most prominent L-ascorbate associated effects are visible in F406S/A407S/Q408P and T405S/F406S/A407S, it seems that the presence of a tandem of Ser residues within the NAT substrate binding site, and the removal of the planar aromatic Phe reside, favor L-ascorbate recognition by the UapA mutants. Importantly, our results show that the L-ascorbate effect on hyphae morphology and the loss of GFP fluorescent are both UapA-specific, as we did not detect the same negative effects in a strain expressing FurA-GFP, which does not express UapA or any other nucleobase transporter (see strain list in Materials and methods). 
a

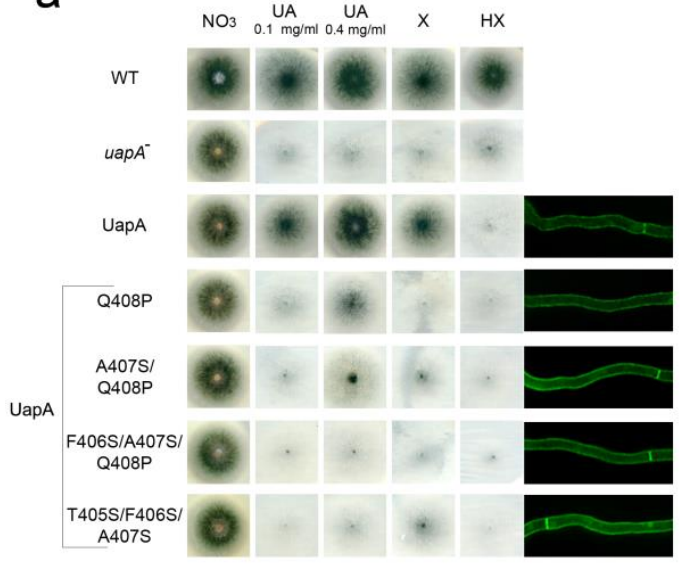

b

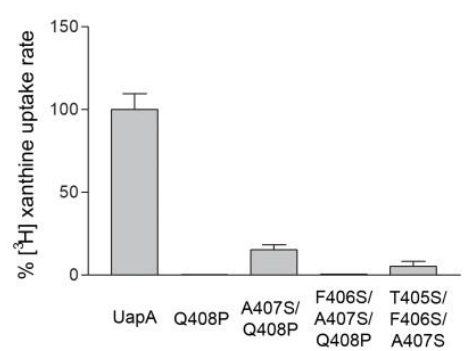

C

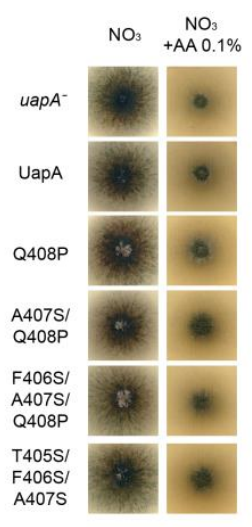

d

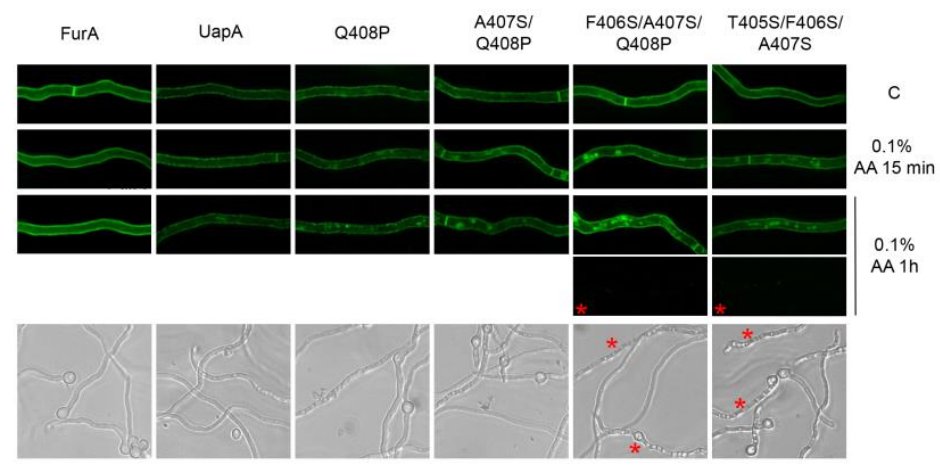

e

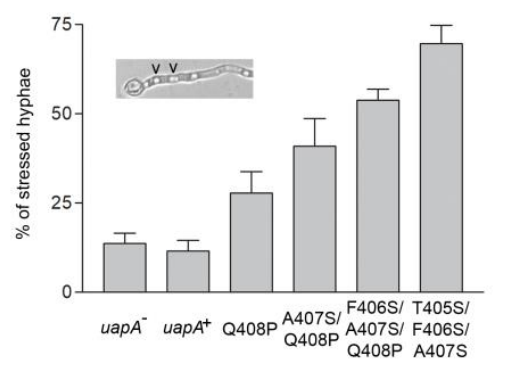

FIGURE 2: Specific residues within the NAT signature motif are critical for UapA function and specificity. (a) Growth tests, on standard MM, of isogenic strains expressing wild-type (UapA) or mutant UapA versions (Q408P, A407S/Q408P, T405S/F406S/A407S and F406S/A407S/Q408P), expressed in a genetic background genetically lacking other major purine transporters (i.e. $\triangle u a p C \triangle a z g A$ ). A strain lacking a functional UapA (uapA-) and a standard wild-type $A$. nidulans strain (WT) are also included in the test as controls. The test depicts growth on $10 \mathrm{mM}$ sodium nitrate $\left(\mathrm{NO}_{3}\right)$ or $0.1 \mathrm{mg} / \mathrm{ml}$ uric acid (UA), xanthine (X), hypoxanthine ( $\left.\mathrm{HX}\right)$, after $48 \mathrm{~h}$ at $37^{\circ} \mathrm{C}$. A $4-$ fold higher concentration of UA $(0.4 \mathrm{mg} / \mathrm{ml})$ was also used in order to distinguish UapA mutants with highly reduced affinity for substrates (Q408P, A407S/Q408P) from mutants with an apparent total loss of transport activity (T405S/F406S/A407S and F406S/A407S/Q408P). Subcellular localization of wild-type or mutant UapA-GFP versions, as revealed by in vivo epifluorescence microscopy, is also shown at the right side of the panel. Notice that in all cases UapA-GFP is stably localized in the plasma membrane and the septa of $A$. nidulans hyphae. For details of sample preparation see Material and methods. (b) Comparative UapA-mediated uptake rates of radiolabeled ${ }^{3} \mathrm{H}$-xanthine in strains expressing wild-type (UapA) or mutant versions (Q408P, A407S/Q408P, T405S/F406S/A407S and F406S/A407S/Q408P) of UapA-GFP. Results are averages of three measurements for each concentration point. SD was $<20 \%$. (c) Growth tests, at $37^{\circ} \mathrm{C}$, on standard MM supplemented with $10 \mathrm{mM}$ sodium nitrate $\left(\mathrm{NO}_{3}\right)$ as nitrogen source in the absence or presence of $0.1 \% \mathrm{~L}$-ascorbate (AA) of isogenic strains lacking UapA (uapA), or expressing wild-type (UapA) or mutant UapA versions (Q408P, A407S/Q408P, T405S/F406S/A407S and F406S/A407S/Q408P). (d) Upper panel: In vivo epifluorescence microscopy following the effect of L-ascorbate (0.1\% AA) on UapA-GFP or FurA-GFP subcellular localization. Notice that after 15 min addition of AA clear endocytic turnover of UapA-GFP (i.e. appearance of cytoplasmic structures corresponding to vacuoles; Gournas et al., 2010) is observed in all UapA mutants, but not in wild-type UapA or FurA. Vacuolar turnover is more evident after $1 \mathrm{~h}$ of AA in the triple mutants T405S/F406S/A407S and especially F406S/A407S/Q408P, where more and larger vacuoles appear. Notice that after $1 \mathrm{~h}$ of $A A$, moderate vacuolar turnover is also evident in the wild-type UapA, but not in FurA. Noticeably also, $1 \mathrm{~h}$ in AA triggers "loss" of GFP fluorescence in a significant fraction (>50\%) of hyphae in in the triple mutants T405S/F406S/A407S and F406S/A407S/Q408P (lowest black boxes marked with a red asterisk). The same effect, albeit significantly reduced, is also seen in A407S/Q408P (36\%) and Q408P (22\%), while in the wild-type UapA-GFP or FurA-GFP fluorescence loss is < 10\% (not shown). Lower panel: L-ascorbate (1\% AA for $1 \mathrm{~h}$ ) triggers differential hypervacuolarization and reduction of hyphal width (i.e. cell stress) in UapA mutants and controls. Stressed hyphae are marked with a red asterisk. (e) Quantification of the effect of L-ascorbate on hyphal morphology, as recorded for 100 hyphae, of each strain analyzed. In all cases, hypervacuolarization and reduction of the width of hyphae was associated with loss of fluorescence (not shown). The results depicted in the graph confirm the apparent cytotoxicity of L-ascorbate in the triple mutants T405S/F406S/A407S and F406S/A407S/Q408P, where 50-70\% of hyphae seem stressed, followed by progressively lower effects on A407S/Q408P (40\%), Q408P (27\%) and wt UapA (11\%). 
UapA can bind L-ascorbate with extremely low affinity: evidence for evolution of L-ascorbate transporters via optimization of a NAT sub-function

The observation that addition of L-ascorbate can also trigger a degree of vacuolarization and endocytic turnover of wild-type UapA-GFP, made us re-examine whether UapA can recognize, even with very low affinity, L-ascorbate. We performed standard competitive inhibition assays to test whether increasing concentrations of L-ascorbate (1-60 mM) inhibit the uptake of radiolabeled ${ }^{3} \mathrm{H}$-xanthine $(1 \mu \mathrm{M})$. Results shown in Figure 3 establish that wild-type UapA can indeed bind L-ascorbate with very low affinity, reflected in a $K_{\mathrm{i}}$ value of $\sim 17 \mathrm{mM}$. We repeated the same experiment using a promiscuous UapA mutant capable of recognizing all nucleobases and obtained a moderately increased binding affinity also for L-ascorbate (Ki $11 \mathrm{mM})$. This mutant (R481G/T526M) has an intact NAT signature motif, but includes two mutations that seem to loosen its specificity by modifying the functioning of putative outward and inward gates [29, 33]. Thus, UapA can in principle bind L-ascorbate with an affinity approx. 1000-fold lower than the one for its physiological substrates (xanthine or uric acid), or and 10- to 100-lower than its affinity for other nucleobase analogues [47]. This finding supports the hypothesis that other nucleobase-specific NATs might also be capable of very low-affinity recognition o L-ascorbate. This in turn supports a model where L-ascorbate NATs evolved via a progressive improvement of a sub-function of nucleobase-specific NATs.

\section{CONCLUSIONS}

Nucleobases are planar molecules with relatively low solubility, whereas L-ascorbate is non-planar and extremely soluble. Such a difference should necessitate prominent changes in the architecture of the substrate-binding site and of the substrate translocation trajectory in the relevant NATs. Our present analysis supports that NAT neo- functionalization took place via progressive improvement of a sub-function (i.e. very low affinity binding of L-ascorbate) in the course of evolution and probably under "new" selective pressures imposed by the evolution of L-ascorbate biosynthesis. This idea leads to many more questions. Are there any present day NATs transporting both substrates equally efficiently? Or are there NATs that can transport L-ascorbate more efficiently than nucleobases? If cases of double specificity exist, could these represent intermediate versions in the evolutionary path from nucleobase to L-ascorbate recognition? Or did only extinct ancestral nucleobase-transporters had this double specificity? NATs that contain transitory variations of NAT signature motif might reflect extant intermediate steps in the evolution of L-ascorbate transporters.

Still many more questions remain to be answered in the direction opened by the present work. Has the specificity shift from nucleobase to L-ascorbate occurred via transporter intermediates that had specificities other than nucleobase or L-ascorbate (could the SVCT3 clade being such a putative case for example?), or even little or no function at all? Can transporters evolve the ability to recognize a new substrate before or after this substrate first appears and how this is related to emergence of substrates via evolution of biosynthetic pathways? Very recently, it was shown that NPF transporters are able to transport both cyanogenic glucosides and glucosinolates, before plants evolved the ability to synthesize glucosinolates [52]. Later in evolution, these dual-specificity transporters specialized to transport only glucosinolates. In addition, Jørgensen et al. [52] also showed that early glucosinolate transporters could move a broad variety of glucosinolates, but later evolved to only transport particular types. These findings support the idea that evolution of transporter specificity and biosynthetic pathways might evolve together. An analogous case concerning the evolution of specificity in vertebrate corticoid receptors was reported earlier

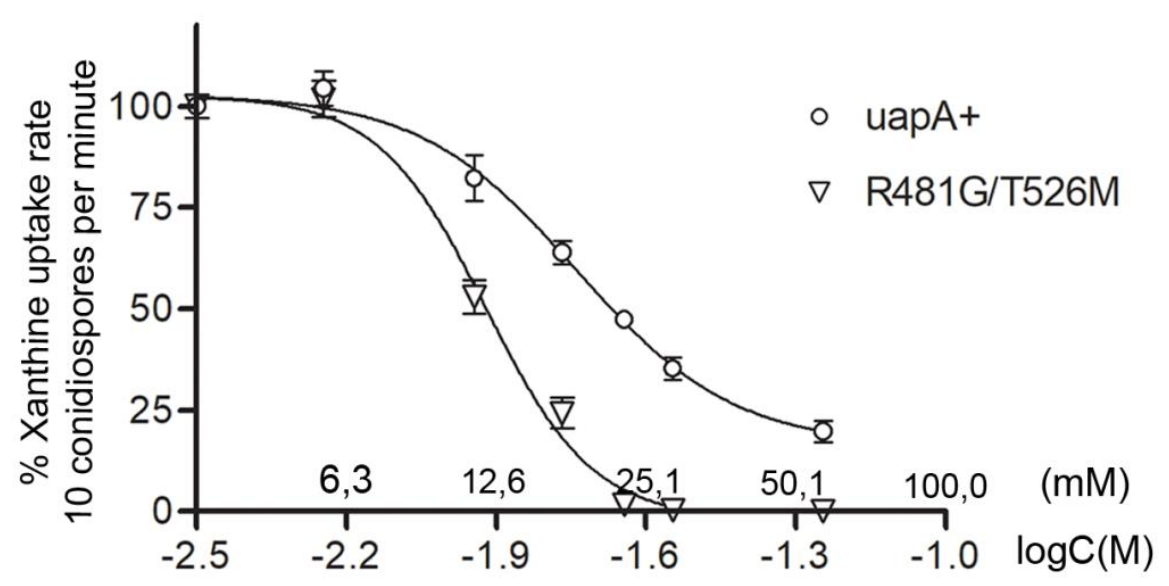

Ascorbic acid concentration
FIGURE 3: L-ascorbate inhibits UapA transport activity. The graph shows dose response curves of $\left[{ }^{3} \mathrm{H}\right]$-xanthine uptake rate in strains expressing either wild-type UapA or the isogenic mutant UapA-R481G/T526M, in the presence of increasing concentration of non-radiolabeled L-ascorbate (0-56 mM). In both cases, L-ascorbate inhibited UapA transport activity, with $K_{\mathrm{i}}$ values estimated at 17 and $11 \mathrm{mM}$ for UapA and UapA-R481G/T526M, respectively. Results are averages of three measurements for each concentration point. SD was $<18 \%$. For details of measurements and $K_{\mathrm{i}}$ estimation see Materials and methods. 
$[53,54]$. Corticoid and glucocorticoid receptors evolved post duplication of a dual specificity aldosterone and cortisol receptor basal to the jawed vertebrate. However, aldosterone biosynthesis did not arise before the advent of tetrapods suggesting that the ancestral receptor evolved affinity towards aldosterone before the hormone was present, possibly as a by-product of the receptors' affinity towards chemically similar ligands [54].

L-ascorbate biosynthesis seems to have evolved in plants and animals (prokaryotes do not synthesize it) via three major routes. The animal kingdom uses one of these pathways, and photosynthetic eukaryotes (plants, algae and some single-celled organisms) employ the other two. All three pathways proceed via different intermediate molecules, but the two from photosynthetic eukaryotes both use an enzyme called galactolactone dehydrogenase (GLDH), instead of gulonolactone oxidase (GULO), to catalyze the final step [55]. Some evidence exists that GULO and GLDH might have evolved from a common ancestor. Interestingly, certain animals, including guinea pigs, most bats, some fish and bird species, as well as humans and many other primates, have lost the ability to synthesize Lascorbate. These animals obtain ascorbate from their diets [55].

Another important gene loss in vertebrates, possibly relative to NAT evolution, concerns purine catabolism. Most animals oxidize purines into allantoin, which is excreted from their body as the end product of purine catabolism. Humans and other primates are among some groups that lost the gene for uric acid oxidase (i.e. present as a pseudogene), which catalyzes of oxidation uric acid to allantoin [56]. This gene loss is partly responsible for the elevated levels of uric acid in blood plasma. Uric acid is a powerful antioxidant and is a scavenger of singlet oxygen and radicals, in a way similar, but not identical, with the action of L-ascorbic acid [57]. It has been argued that the loss of uric acid oxidase and elevation of uric acid levels in the body have played an important role in the development of the primate brain and human intelligence due to its protective role against oxidation related to neurotoxic activity. High uric acid levels have also been linked to human life span $[57,58]$. Could it be that evolution of NAT specificity, which shifted from one outstanding antioxidant (uric acid) to another (L-ascorbic acid), and the loss of nucleobase-specific NATs in primates, be related to the coevolution of relevant metabolic pathways? Relative to such questions, recent evidence supported the co-evolution of the uric acid transporter URAT1 (distinct from NAT transporters) and uric acid oxidase during primate evolution [59]. To most of the above questions, a future challenge will be to understand the detailed molecular changes that led to changes in specificity and possibly relate their outcome to the evolution of relative metabolic pathways.

\section{MATERIALS AND METHODS}

\section{Media, growth conditions and strains}

Standard media for A. nidulans were used. Media and supplemented auxotrophies were at the concentrations given in the Fungal Genetics Stock Center database (http://www.fgsc.net). Nitrogen sources were used at final concentrations of $10 \mathrm{mM}$ sodium nitrate or $0.5 \mathrm{mM}$ of purines. A. nidulans strains used are listed in Supplementary Table S1. The recipient for transformations was the uapA $\triangle$ uapC $\triangle$ azgA $\triangle$ pabaA1 argB2 mutant strain lacking the endogenous uapA gene, as well as the genes encoding a secondary xanthine/uric acid transporter (UapC) and the major purine transporter AzgA. Plasmids carrying gfp-tagged wild-type (wt) or mutant versions of uapA (i.e. UapA-GFP, [60]) were introduced by standard genetic transformation into the recipient strain and transformants were selected on the basis of the $\arg B$ arginine auxotrophy ( $\arg B 2)$ complementation [61]. The strain expressing FurA-GFP, used as a control in Figure 2D, is described in [61]. GFP C-terminal tagging has previously shown to have absolutely no effect on UapA or FurA transport kinetics [51].

\section{Standard nucleic acid manipulations}

Genomic DNA extraction from A. nidulans was as described in http://www.fgsc.net. Plasmid preparation from E. coli strains and DNA bands were purified from agarose gels were done with the Nucleospin Plasmid kit and the Nucleospin Extractll kit according to the manufacturer's instructions (MachereyNagel, Lab Supplies Scientific SA, Hellas). Restriction enzymes were from Takara Bio (Lab Supplies Scientific SA, Hellas). Conventional PCR reactions were done with KAPA-Taq DNA polymerase (Kapa Biosystems, Lab Supplies Sci- entific SA, Hellas). Cloning and amplification of products were done with Kapa HiFi (Kapa Biosystems, Lab Supplies Scientific SA, Hellas).

\section{Construction and analysis of Aspergillus mutants}

All uapA mutations appearing in this work were generated by standard site-directed mutagenesis, using specific oligonucleotides (Supplementary Table S2), the QuikChange Mutagenesis Kit and plasmid pAN510-GFP carrying a gfp-tagged uapA gene, as a template $[47,60]$. The Q408P mutant was constructed previously $[27,47]$. Mutant alleles of uapA were introduced by genetic transformation into the recipient strain uapAD uapCD $a z g A D$ pabaA1 argB2. Selected transformants were purified and those arising from single-copy plasmid integration, as evidenced by PCR and Southern blot analysis, identified [61]. Transformants were tested, at 25 or $37^{\circ} \mathrm{C}, \mathrm{pH} 6.8$, for their ability to grow on $\mathrm{MM}$ supplemented with uric acid or other purines as nitrogen sources, or for their general growth phenotype in the presence of L-ascorbate (concentration range from 0.1 to $1 \%$ ). Transformants were also tested, using epifluorescence inverted microscopy (using a Zeiss Observer Z1/AxioCam HR R3 camera/Zen lite 2012 software) in respect to UapA-GFP subcellular localization, both in the absence or presence of L-ascorbate $(0.01-0.2 \%)$ for 15,60 or $120 \mathrm{~min}$. Samples for epifluorescence microscopy were prepared as previously described, after 16-18 h of growth in standard MM supplements with sodium nitrate as nitrogen source [34].

\section{Transport assays}

UapA mutants were functionally characterized by uptake assays performed in living cells. Radiolabelled $\left[{ }^{3} \mathrm{H}\right]$-xanthine (22.8 $\mathrm{Ci} \mathrm{mmol}^{-1}$, Moravek Biochemicals, CA, USA) uptake measurements were performed with $A$. nidulans germinating conidiospores concentrated at $10^{7}$ conidiospores per $100 \mathrm{ml}$ at $37^{\circ} \mathrm{C}, \mathrm{pH} 6.8$, at the end of the isotropic growth phase, in which UapA shows maximal expression [62]. Competition experiments of $\left[{ }^{3} \mathrm{H}\right]$-xanthine uptake were carried out with in- 
creasing concentrations of unlabeled L-ascrobic acid (1-60 mM). All transport assays were carried out in at least three independent experiments, with three replicates for each concentration or time point and s.d. was $<20 \%$ in all cases.

\section{Phylogenetic analysis datasets and methods}

We selected representative NAT sequences from organisms across all domains of life, representing all major taxa, with particular emphasis in characterized family members of known specificity. For the trees shown in Figure 1 and Supplementary Figure S2, which address in detail the plant and animal NATs (SVCT clades) and the evolution of L-ascorbate transporters, we started by detecting NAT domain containing sequences in complete sequenced genomes, coming from Ensembl release 91, Ensembl Metazoa release 38, and selected species from Ensembl Plants release 38. To detect NAT sequences in the genomes we used HMMER and the PF00860 (Xan_ur_permease) Pfam HMM profile, and performed an hmmsearch using the GA (gathering) curated threshold (cut_ga parameter). For the 728 detected sequences, a multiple sequence alignment was built using MAFFT v6.861b in the L-INS-i mode, designed for "set of sequences containing sequences flanking around one alignable domain". The resulting alignment was trimmed using trimAl v1.3 [63] using a gap score cut-off of 0.1 . The best-fit evolutionary model selection was selected prior to the phylogenetic inference using ProtTest 3 [64]. Three different evolutionary models were tested (JTT, WAG and LG). The model best fitting the data was determined by comparing the likelihood of all models according to the AIC criterion [65]. An ML tree was inferred with RAxML v8.2.11 [66] using the best-fitting model. A discrete gammadistribution model with four rate categories plus invariant positions was used. The gamma parameter and the fraction of invariant positions were estimated from the data. Branch support was computed using an aLRT (approximate likelihood ratio test). We used the exact same methods for the phylogenetic tree of only the SVCT clade, a subset of the previous tree, consisting of 674 sequences, apart from the MAFFT algorithm used, which was the G-INS-I in this case (assumes that entire region can be aligned). ETE toolkit [42] was used for the execution of the phylogenetic pipelines and the visualization of all phylogenetic trees in Figure 1 and Supplementary Figures S1

\section{REFERENCES}

1. Ohno S (1970). Evolution by gene duplication. Springer-Verlag, Berlin Heidelberg. doi: 10.1007/978-3-642-86659-3

2. Taylor JS, and Raes J. (2004). Duplication and divergence: the evolution of new genes and old ideas. Annu Rev Genet 38:615-643. doi: 10.1146/annurev.genet.38.072902.092831

3. Bergthorsson U, Andersson DI, and Roth JR (2007). Ohno's dilemma: Evolution of new genes under continuous selection. Proc Natl Acad Sci USA 104 (43): 17004-17009. doi: 10.1073/pnas.0707158104

4. Bershtein S, and Tawfik DS (2008). Ohno's model revisited: measuring the frequency of potentially adaptive mutations under various mutational drifts. Mol Biol Evol 25(11):2311-2318. doi: 10.1093/molbev/msn174

5. Lynch $\mathrm{M}$, and Conery JS (2000). The evolutionary fate and consequences of duplicate genes. Science 290 (5494):1151-5. doi: 10.1126/science.290.5494.1151 and S2. ETE was also used for plotting the NCBI taxonomy tree for the 171 species, and the frequency profile of the subfamilies (Fig. 1). For the tree shown in Supplementary Figure S1, which includes all functionally characterized NATs from bacteria and fungi and their closest homologues from other prokaryotic and eukaryotic microorganisms, we obtained the relative sequences by blastP (https://blast.ncbi.nlm.nih.gov) and performed a multiple sequence alignment with MUSCLE v7.0.26 (http://www.megasoftware.net/). MEGA was used for testing the aligned sequences for optimal amino acid substitution model. According to the AIC, the LG+G+F model [67] was selected and the tree was created using a maximum likelihood (ML), and visualized by FigTree v1.4.3 (http://tree.bio.ed.ac.uk/software/figtree/).

\section{ACKNOWLEDGEMENTS}

We thank loannis Theologidis for critical assessment of the phylogenetic analysis presented herein. This work was supported by a "Stavros Niarchos Foundation" research grant.

\section{SUPPLEMENTAL MATERIAL}

All supplemental data for this article are available online at www.microbialcell.com.

\section{CONFLICT OF INTEREST}

The authors declare no conflict of interest.

\section{COPYRIGHT}

(C) 2018 Kourkoulou et al. This is an open-access article released under the terms of the Creative Commons Attribution (CC BY) license, which allows the unrestricted use, distribution, and reproduction in any medium, provided the original author and source are acknowledged.

Please cite this article as: Anezia Kourkoulou, Alexandros A. Pittis and George Diallinas (2018 Evolution of substrate specificity in the Nucleobase-Ascorbate Transporter (NAT) protein family. Microbial Cell 5(6): 280-292. doi: 10.15698/mic2018.06.636

6. Force A, Lynch M, Pickett FB, Amores A, Yan YL, and Postlethwait J (1999). Preservation of duplicate genes by complementary, degenerative mutations. Genetics 151(4):1531-1545. PMID: 10101175

7. Grauer D, and Wen-Hsiung Li (2000). Fundamentals of molecular evolution. Sunderland, MA. Sinauer. pp 282-283. doi: 10.1002/jctb. 375

8. Hittinger CT, and Carroll SB (2007). Gene duplication and the adaptive evolution of a classic genetic switch. Nature 449 (7163): 677-681. doi: 10.1038/nature06151

9. Averof M. (2002). Arthropod Hox genes: insights on the evolutionary forces that shape gene functions. Curr Opin Genet Dev 12(4):386392. doi: 10.1016/s0959-437x(02)00314-3

10. Tokuriki N, and Tawfik DS (2009). Protein dynamism and evolvability. Science 324(5924):203-207. doi: 10.1126/science.1169375 
11. Soskine M, and Tawfik DS (2010). Mutational effects and the evolution of new protein functions. Nat Rev Genet 11(8):572-582. doi: $10.1038 / \mathrm{nrg} 2808$

12. Goldsmith $M$, and Tawfik DS (2012). Directed enzyme evolution: beyond the low-hanging fruit. Curr Opin Struct Biol 22(4):406-412. doi: 10.1016/j.sbi.2012.03.010

13. Starr TN, and Thornton JW (2016). Epistasis in protein evolution. Protein Sci 25(7): 1204-1218. doi: 10.1002/pro.2897

14. Hochberg GKA, and Thornton JW (2017). Reconstructing Ancient Proteins to Understand the Causes of Structure and Function. Annu Rev Biophys 46:247-269. doi: 10.1146/annurev-biophys-070816033631

15. Khersonsky O, and Tawfik DS (2010). Enzyme promiscuity: a mechanistic and evolutionary perspective. Annu Rev Biochem79:471-505. doi: 10.1146/annurev-biochem-030409-143718

16. Nyathi $Y$, Wilkinson BM, and Pool MR (2013). Co-translational targeting and translocation of proteins to the endoplasmic reticulum. Biochim Biophys Acta 1833(11): 2392-402. doi: 10.1016/j.bbamcr.2013.02.021

17. Foot $\mathrm{N}$, Henshall $\mathrm{T}$, and Kumar S (2017). Ubiquitination and the Regulation of Membrane Proteins. Physiol Rev 97(1):253-281. PMID: 27932395

18. MacGurn JA, Hsu PC, and Emr SD (2012). Ubiquitin and membrane protein turnover: from cradle to grave. Annu Rev Biochem 81:231259. doi: 10.1146/annurev-biochem-060210-093619

19. Gournas C, Papageorgiou I, and Diallinas G (2008). The nucleobase-ascorbate transporter (NAT) family: genomics, evolution, structure-function relationships and physiological role. Mol Biosyst 4(5):404-416. doi: 10.1039/b719777b

20. Diallinas G, and Gournas C (2008). Structure-function relationships in the nucleobase-ascorbate transporter (NAT) family: lessons from model microbial genetic systems. Channels (Austin) 2(5):363-372. PMID: 18981714

21. Dialinas G (2016). Dissection of Transporter Function: From Genetics to Structure. Trends Genet 32(9):576-590. doi: 10.1016/j.tig.2016.06.003

22. Frillingos $S$ (2012). Insights to the evolution of nucleobaseascorbate transporters (NAT/NCS2 family) from the Cys-scanning analysis of xanthine permease XanQ. Int J Biochem Mol Biol 3:250272. PMID: 23097742

23. Bürzle $M$, Suzuki $Y$, Ackermann D, Miyazaki $H$, Maeda $N$, Clémençon $B$, Burrier $R$, and Hediger MA (2013). The sodiumdependent ascorbic acid transporter family SLC23. Mol Aspects Med 34(2-3):436-454. doi: 10.1016/j.mam.2012.12.002

24. Argyrou E, Sophianopoulou V, Schultes N, and Diallinas G (2001). Functional characterization of a maize purine transporter by expression in Aspergillus nidulans. Plant Cell 13(4):953-964. PMID: 11283348

25. Niopek-Witz S, Deppe J, Lemieux MJ, and Möhlmann T (2014). Biochemical characterization and structure-function relationship of two plant NCS2 proteins, the nucleobase transporters NAT3 and NAT12 from Arabidopsis thaliana. Biochim Biophys Acta 1838(12):3025-3035. doi: 10.1016/j.bbamem.2014.08.013

26. Amillis S, Koukaki M, and Diallinas G (2001) Substitution F569S converts UapA, a specific uric acid-xanthine transporter, into a broad specificity transporter for purine-related solutes. J Mol Biol 313:765774. doi: 10.1006/jmbi.2001.5087

27. Papageorgiou I, Gournas C, Vlanti A, Amillis S, Pantazopoulou A, and Diallinas $G$ (2008). Specific interdomain synergy in the UapA transporter determines its unique specificity for uric acid among NAT carriers. J Mol Biol 382(5):1121-1135. doi: 10.1016/j.jmb.2008.08.005
28. Vlanti A, Amillis S, Koukaki M, and Diallinas G (2006). A novel-type substrate-selectivity filter and ER-exit determinants in the UapA purine transporter. J Mol Biol 357(3):808-819. doi: 10.1016/j.jmb.2005.12.070

29. Kosti V, Papageorgiou I, and Diallinas G (2010). Dynamic elements at both cytoplasmically and extracellularly facing sides of the UapA transporter selectively control the accessibility of substrates to their translocation pathway. J Mol Biol 397(5):1132-1143. doi: 10.1016/j.jmb.2010.02.037

30. Västermark A, and Saier MH Jr (2014). Evolutionary relationship between $5+5$ and $7+7$ inverted repeat folds within the amino acidpolyamine-organocation superfamily. Proteins 82(2):336-346. doi: 10.1002/prot.24401

31. Yamamoto S, Inoue K, Murata T, Kamigaso S, Yasujima T, Maeda JY, Yukihiro Yoshida J, Ohta K, and Yuasa H (2010). Identification and Functional Characterization of the First Nucleobase Transporter in Mammals. J Biol Chem 285(9): 6522-6531. doi: 10.1074/jbc.M109.032961

32. Lu F, Li S, Jiang $Y$, Jiang J, Fan H, Lu G, Deng D, Dang S, Zhang X, Wang J, and Yan $N$ (2011). Structure and mechanism of the uracil transporter UraA. Nature 14;472(7342):243-246. doi: 10.1038/nature09885

33. Alguel Y, Amillis S, Leung J, Lambrinidis G, Capaldi S, Scull NJ, Craven $G$, Iwata $S$, Armstrong A, Mikros E, Diallinas $G$, Cameron AD, and Byrne B (2016). Structure of eukaryotic purine/H(+) symporter UapA suggests a role for homodimerization in transport activity. Nat Commun 7:11336. doi: $10.1038 /$ ncomms11336

34. Martzoukou O, Karachaliou M, Yalelis V, Leung J, Byrne B, Amillis S and Diallinas $G$ (2015). Oligomerization of the UapA purine transporter is critical for ER-exit, plasma membrane localization and turnover. J Mol Biol 427(16):2679-2696. doi: 10.1016/j.jmb.2015.05.021

35. Yu X, Yang G, Yan C, Baylon JL, Jiang J, Fan H, Lu G, Hasegawa K, Okumura H, Wang T, Tajkhorshid E, Li S, and Yan N (2017). Dimeric structure of the uracil:proton symporter UraA provides mechanistic insights into the SLC4/23/26 transporters. Cell Res 27(8):1020-1033. doi: $10.1038 / c r .2017 .83$

36. Krypotou E, Lambrinidis G, Evangelidis T, Mikros E, and Diallinas G (2014). Modelling, substrate docking and mutational analysis identify residues essential for function and specificity of the major fungal purine transporter AzgA. Mol Microbiol 93(1):129-145. doi: 10.1111/mmi.12646

37. Coudray N, L Seyler S, Lasala R, Zhang Z, Clark KM, Dumont ME, Rohou A, Beckstein O, and Stokes DL (2007). Structure of the SLC4 transporter Bor1p in an inward-facing conformation. Protein Sci 26(1):130-145. doi: 10.1002/pro.3061

38. Arakawa T, Kobayashi-Yurugi T, Alguel $Y$, Iwanari $H$, Hatae $H$, Iwata M, Abe Y, Hino T, Ikeda-Suno C, Kuma H, Kang D, Murata T, Hamakubo $\mathrm{T}$, Cameron AD, Kobayashi T, Hamasaki N, and Iwata S (2015). Crystal structure of the anion exchanger domain of human erythrocyte band 3. Science. 350(6261):680-684. doi: 10.1126/science.aaa4335

39. Geertsma ER, Chang YN, Shaik FR, Neldner Y, Pardon E, Steyaert J, and Dutzler R (2015). Structure of a prokaryotic fumarate transporter reveals the architecture of the SLC26 family. Nat Struct Mol Biol 22(10):803-808. doi: 10.1038/nsmb.3091

40. Byrne B (2017). It takes two to transport via an elevator. Cell Research 27:965-966. doi: 10.1038/cr.2017.89

41. Wong S, and Wolfe KH (2005). Birth of a metabolic gene cluster in yeast by adaptive gene relocation. Nat Genet 37(7):777-782. doi: 10.1038/ng1584 
42. Huerta-Cepas J, Serra F, Bork P (2016). ETE 3: Reconstruction, Analysis, and Visualization of Phylogenomic Data. Mol Biol Evol 33(6):1635-1638. doi: 10.1093/molbev/msw046

43. Eddy SR (2011). Accelerated Profile HMM Searches. PLoS Comput Biol. 7(10):e1002195. doi: 10.1371/journal.pcbi.1002195

44. Krypotou E, Scazzocchio C, and Diallinas G (2015). Functional characterization of NAT/NCS2 proteins of Aspergillus brasiliensis reveals a genuine xanthine-uric acid transporter and an intrinsically misfolded polypeptide. Fungal Genet Biol 75:56-63. doi: 10.1016/j.fgb.2015.01.009

45. Pantazopoulou A, and Diallinas G (2006). The first transmembrane segment (TMS1) of UapA contains determinants necessary for expression in the plasma membrane and purine transport. Mol Membr Biol 23(4):337-348. doi: 10.1080/09687860600738239

46. Kosti V, Lambrinidis G, Myrianthopoulos V, Diallinas G, and Mikros $E$ (2012). Identification of the substrate recognition and transport pathway in a eukaryotic member of the nucleobase-ascorbate transporter (NAT) family. PLoS One 7(7):e41939. doi: 10.1371/journal.pone.0041939

47. Koukaki M, Vlanti A, Goudela S, Pantazopoulou A, Gioule H, Tournaviti $S$, and Diallinas $G$ (2005). The nucleobase-ascorbate transporter (NAT) signature motif in UapA defines the function of the purine translocation pathway. J Mol Biol 350(3):499-513. doi: 10.3410/f.1026664.323301

48. Karatza P, Panos P, Georgopoulou E, and Frillingos S (2006). Cysteine-scanning analysis of the nucleobase-ascorbate transporter signature motif in YgfO permease of Escherichia coli: Gln-324 and Asn-325 are essential and Ile-329-Val-339 form an alpha-helix. J Biol Chem 281(52):39881-39890. doi: 10.1074/jbc.m605748200

49. Gournas C, Amillis S, Vlanti A, and Diallinas G (2010). Transportdependent endocytosis and turnover of a uric acid-xanthine permease. Mol Microbiol 75(1):246-260. doi: 10.1111/j.1365-2958.2009.06997.x

50. Gournas C, Prévost M, Krammer EM, and André B (2016). Function and Regulation of Fungal Amino Acid Transporters: Insights from Predicted Structure. Adv Exp Med Biol 892:69-106. doi: 10.1007/978-3319-25304-6_4

51. Krypotou E, Evangelidis T, Bobonis J, Pittis AA, Gabaldón T, Scazzocchio C, Mikros E, Diallinas G (2015). Origin, diversification and substrate specificity in the family of NCS1/FUR transporters. Mol Microbiol 96(5):927-950. doi: 10.1111/mmi.12982

52. Jørgensen ME, Xu D, Crocoll C, Ramırez D, Motawia M S, Olsen C E, Nour-Eldin $\mathrm{HH}$, and Halkier BA (2017). Origin and evolution of transporter substrate specificity within the NPF family. eLife 6:1-30. doi: 10.7554/eLife.19466

53. Bridgham JT, Ortlund EA, and Thornton JW (2009). An epistatic ratchet constrains the direction of glucocorticoid receptor evolution. Nature 461(7263):515-519. doi: 10.1038/nature08249

54. Carroll SM, Bridgham JT, and Thornton J C (2008). Evolution of Hormone Signaling in Elasmobranchs by Exploitation of Promiscuous Receptors. Mol Biol Evol 25(12): 2643-2652. doi: 10.1093/molbev/msn204
55. Wheeler G, Ishikawa T, Pornsaksit V, and Smirnoff N (2015). Evolution of alternative biosynthetic pathways for vitamin $C$ following plastid acquisition in photosynthetic eukaryotes. Elife 13:4. doi: 10.7554/eLife.06369

56. Oda M, Satta Y, Takenaka O, and Takahata N (2002). Loss of urate oxidase activity in hominoids and its evolutionary implications. Mo Biol Evol 19(5):640-653. doi: 10.1093/oxfordjournals.molbev.a004123

57. Ames BN, Cathcart R, Schwiers E, and Hochstein P (1981). Uric acid provides an antioxidant defense in humans against oxidant- and radical-caused aging and cancer: a hypothesis. Proc Natl Acad Sci USA. 78(11):6858-6862. PMID: 6947260

58. Álvarez-Lario B, Macarrón-Vicente J (2010). Uric acid and evolution. Rheumatology (Oxford) 49(11):2010-2015. doi 10.1093/rheumatology/keq204

59. Tan PK, Farrar JE, Gaucher EA, and Miner JN (2016). Coevolution of URAT1 and Uricaseduring Primate Evolution: Implications for Serum Urate Homeostasis and Gout. Mol Biol Evol 33(9):2193-200. doi: 10.1093/molbev/msw116

60. Pantazopoulou A, Lemuh ND, Hatzinikolaou DG, Drevet C, Cecchetto G, Scazzocchio C, and Diallinas G (2007). Differential physiological and developmental expression of the UapA and AzgA purine transporters in Aspergillus nidulans. Fungal Genet Biol 44(7):627-640. doi: 10.1016/j.fgb.2006.10.003

61. Koukaki M, Giannoutsou E, Karagouni A, and Diallinas G (2003). A novel improved method for Aspergillus nidulans transformation. J Microbiol Methods 55(3):687-695. doi: 10.1016/s01677012(03)00208-2

62. Krypotou $E$, and Diallinas $G$ (2014). Transport assays in filamentous fungi: kinetic characterization of the UapC purine transporter of Aspergillus nidulans. Fungal Genet Biol 63:1-8. doi: 10.1016/j.fgb.2013.12.004

63. Capella-Gutiérrez S, Silla-Martínez JM, Gabaldón T (2009). trimAl: a tool for automated alignment trimming in large-scale phylogenetic

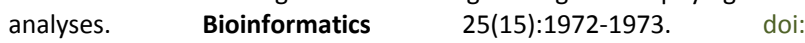
10.1093/bioinformatics/btp348

64. Darriba D, Taboada GL, Doallo R, Posada D (2011). ProtTest 3: fast selection of best-fit models of protein evolution. Bioinformatics 27(8):1164-1165. doi: 10.1093/bioinformatics/btr088

65. Wagenmakers EJ, Farrell S (2004). AIC model selection using Akaike weights. Psychon Bull Rev 11(1):192-196. doi: 10.3758/bf03206482

66. Stamatakis A. (2015) Using RAxML to Infer Phylogenies. Curr Protoc Bioinformatics 51:6.14.1-14. doi: 10.1002/0471250953.bi0614s51

67. Le SQ, Gascuel O (2008). An improved general amino acid replacement matrix. Mol Biol Evol 25(7):1307-1320. doi: 10.1093/molbev/msn067 